\title{
ZEEMAN DOPPLER IMAGING
}

\author{
William Wehlau \\ Department of Astronomy, University of Western Ontario \\ London, Ontario NOM 1Z0, Canada
}

John Rice

Department of Physics and Astronomy, Brandon University

Brandon, Manitoba R7A 6A9, Canada

\begin{abstract}
The mapping of stellar surfaces using the observed profiles of spectral lines is described. The problem is expressed in terms of an integral equation to be solved, using Tikhonov's method. The local line profiles may be given either by an analytic approximation or by the solution of the equation of transfer with a model atmosphere. The model atmospheres commonly used may not correctly represent the stellar atmosphere which may lead to errors in the derived surface maps. Tests of mapping programs and applications to real stars show the capabilities and limitations of surface mapping. Mapping stellar magnetic fields places more severe demands on the data and computational programs than mapping the abundance distributions.
\end{abstract}

\section{INTRODUCTION}

Magnetic fields, inhomogeneous distributions of the chemical elements and the temperature on the surface of a star can be detected and mapped by studying the effects they have on the observed spectra. This was first recognized by Armin Deutsch, who published maps of the magnetic field and distribution of the equivalent widths of some elements on the the surface of HD 125248 in 1958. Since then various names have been applied to this basic procedure, especially if some particular application is emphasized: Surface Mapping, Doppler Imaging, Stellar Tomography, etc.

The mapping procedure relies on two properties to locate a feature on the stellar surface in longitude and latitude: the Doppler effect, and the length of time that a feature contributes to the observed spectra as the star rotates. This implies that mapping cannot be done when the observer is on the axis of rotation of the star. When the observer is in the plane of the star's equator, so that all surface features are visible for one- half the period of rotation, it is impossible to distinguish features in one hemisphere from those in the other. For intermediate inclinations of the axis of rotation, both the longitude and latitude can be obtained, however the latitude of a feature near the equator is not well determined. Generally the most accurately determined part of the 
map lies around the subsolar latitude.

The first maps of stellar surfaces (Deutsch 1958, Pyper 1969, Rice 1970) were restricted by the quality of the photographic spectra and the computational facilities available. Equivalent widths and radial velocities were used as data to derive maps of the magnetic field and local equivalent widths expressed in terms of spherical harmonics. Falk and Wehlau (1974) used more of the information contained in the line profile by representing the profiles by Gaussian curves.

The modern formulation of the problem was first given in 1976 and 1977 when Khokhlova stated the problem as an integral equation, and Goncharsky et al. used Tikhonov's method to solve the inverse problem while making use of all the data in the line profile. They produced maps of the equivalent widths for absorption lines of several elements, although their maps were still limited by the quality of the data and the computer available. Subsequently various authors have produced a number of versions of the mapping problem, some derived from this approach and others independently conceived.

Several reviews of surface mapping have been published in recent years. Consideration of certain technical points are found in the review by Piskunov and Rice (1992). The review by Khokhlova (1985) considers much of the work prior to 1985 and gives an extensive list of references.

Most mapping of the surfaces of Ap stars has concentrated on the distribution of the chemical elements. It has been generally assumed that the inhomogeneous distributions of the elements arise from a stellar magnetic field and that these distributions indicate the structure of the magnetic field. Mapping of stellar magnetic fields using the Zeeman effect has been carried out by Landstreet et al. (1989) and by Brown et al. (1991). It appears likely that many more stars will soon have their magnetic fields mapped by using the Zeeman effect and the circular and linear polarization in their spectral lines. Here, we will first review the mapping of the chemical elements and then consider the problem of mapping the magnetic field by making use of measurements of the polarization in spectral lines.

\section{THE INTEGRAL EQUATION}

The observed spectra represent an integration of the local spectra produced at each point on the visible hemisphere of the star. Each local spectrum is shifted by the Doppler effect corresponding to the radial velocity due to the rotation of the star. This can be expressed by the equation:

$$
R_{o b s}^{*}(\lambda, \phi)=\frac{\iint I_{l}\left[M, \theta, \lambda+\Delta_{D}(M, \phi)\right] \cos \theta d M}{\iint I_{c}(M, \theta) \cos \theta d M}
$$

where
$R_{c b b s}^{*}(\lambda, \phi)$ is the residual intensity of the line that is observed at phase $\phi$
$I_{c}, I_{l} \quad$ are the intensities of the continuum and the line
$M \quad$ is the position on the star specified by latitude and longitude
$\theta \quad$ is the angle between the normal at $M$ and the line of sight
$\Delta_{D}(M, \phi)$ is the Doppler shift at $M$ due to the rotation of the star 
The left side of this equation contains the observed profiles of a spectral line at a number of different phases, usually about ten to twenty. From this equation we wish to derive, as a function of position on the star, whatever is assumed to be responsible for variation of the local line profile - the magnetic field, the abundance of the element, or the temperature. This is done by iteration. An initial distribution is assumed, the local line profiles are calculated and a predicted line profile, $R_{c a l c}^{*}$, is calculated from the equation above. The conjugate-gradient minimization technique is used to adjust the distribution on the surface so as to reduce the discrepancy between the observed and calculated line profiles. This is repeated until the discrepancies between the observed and computed profiles are reduced to a level consistent with the accuracy of the data, or until successive iterations produce no change.

Errors in the data can produce large fluctuations in the derived map over small distances. To control these errors and produce a smoother map, more representative of the actual distribution on the star, a penalty function is introduced, as developed by Tikhonov. The penalty function usually adopted is either Tikonov's functional or Maximum Entropy. Figure 1 shows a map of the distribution of iron on $\epsilon$ UMa produced by the procedure described above (Rice and Wehlau 1990, 1991).

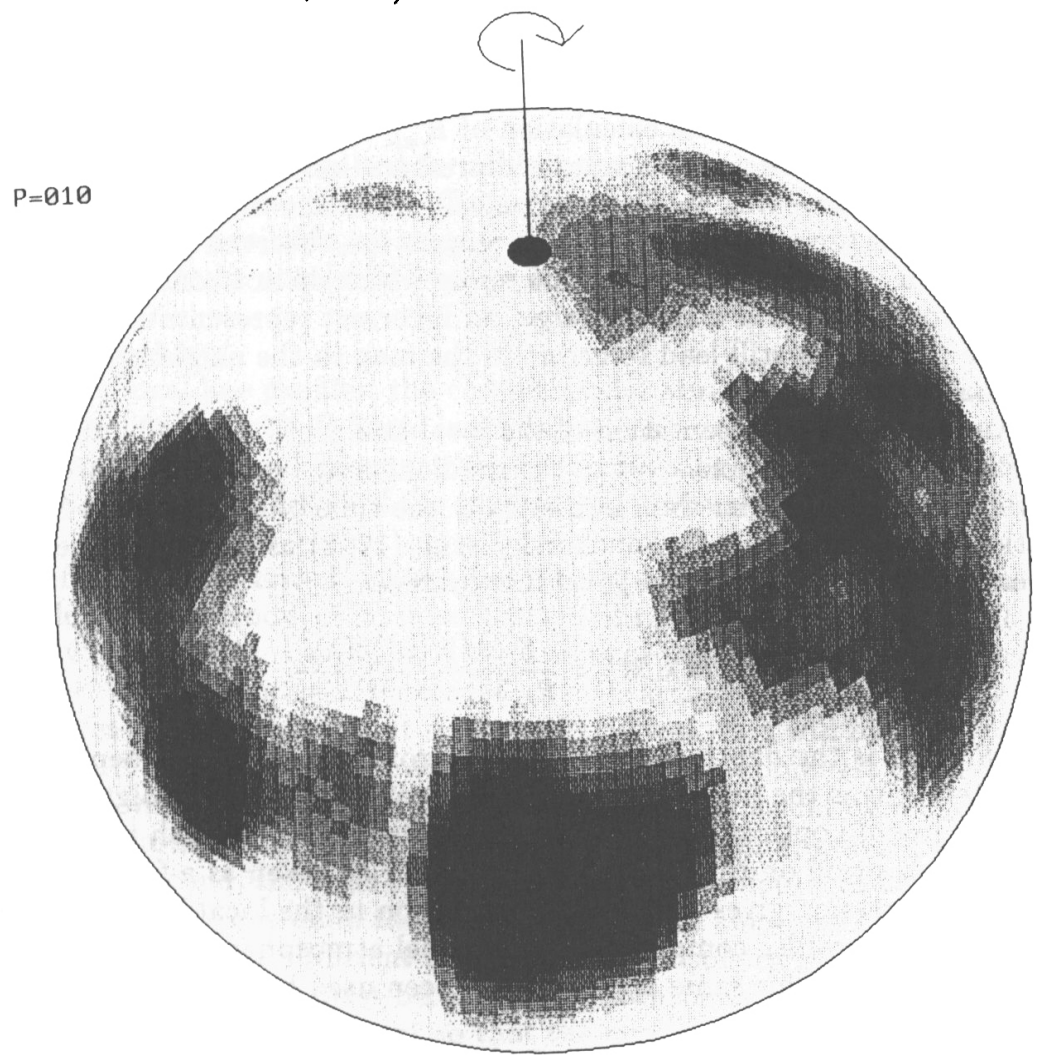

Fig. 1 The distribution of iron on $\epsilon$ UMa. 
In the integral equation various parameters may be regarded as known from other sources or may be regarded as unknowns to be obtained from the solution of the equation. For example, the value of the inclination of the axis of rotation $i$ may have been obtained from the value of $\mathrm{v} \sin i$ and the equatorial velocity, or it may be obtained by solving the integral equation for different values of $i$ and adopting the value which minimizes the final discrepancy between the observed and computed profiles. The evaluation of several parameters is illustrated for 21 Per by Wehlau et al. (1991).

Alternate formulations of mapping problem such as the one described in the paper on Doppler Imaging by Vogt, Penrod, Hatzes (1987) have been given, but they must deal with the same, or equivalent, quantities. Some of these mapping programs solve a more restricted problem, such as the case when the local line profile is regarded as fixed in shape with only the equivalent width of the line differing from one point on the star to another. Here we will consider the more general problem, concentrating on the work of the Russian and Canadian groups.

\section{INPUT PHYSICS}

\section{The Local Line Profile}

In many cases the correct solution of the mapping problem requires that the local line profiles used in the calculation of $R_{c: a l c}^{*}$ at the completion of the iteration procedure be a very accurate representation of the actual local line profiles on the star. This applies particularly when the rotational broadening is small so that the local line profiles dominate the observed profile. Even if the spectrum variability is due to dark spots which do not contribute to the observed spectrum, or if $\mathrm{v} \sin i$ is large, an incorrect representation of the local line profile can still lead to errors in the map in the neighborhood of the rotational pole.

An analytical representation of the local line profiles considerably simplifies the computations. When the gradient may be calculated from an analytic expression rather than numerically the time of computation for each iteration is greatly reduced. Goncharsky et al. (1982) used the Minnaert (1935) approximation to represent the local line profiles:

$$
R(\lambda, M)=\frac{R_{s}(M) \tau(M) k(\lambda)}{R_{c}(M)+\tau(M) k(\lambda)}
$$

where $R(\lambda, M)$ is the depth of the local line profile, $R_{c}(M)$ is the depth at the line center, $k(\lambda)$ is the line absorption coefficient given by the Voigt profile, and $\tau$ is a pseudo optical depth at the line center which we wish to obtain as a function of position on the stellar surface. With $R_{c}$ taken as a function of $\tau$, the Minnaert expression gives a good approximation to the local line profile when compared with profiles computed from a model atmosphere (Pavolova and Khokhlova 1980). This same approach has been used by Wehlau et al. (1982) and others subsequently.

Most mapping programs now calculate the local line profiles by numerical integration of the equation of transfer through a model atmosphere. This will increase the computation time considerably if a new local profile is calculated 
at each point on the star for each iteration. A substantial saving can be achieved by first calculating line profiles for light emerging at a number of different angles to the stellar surface and for a number of different values of the abundance of the element (or temperature for later type stars with spots). Interpolation in tables of the profiles to obtain the one required proceeds much more rapidly than the calculation of the profiles themselves. The CPU time required will, of course, depend on the number of phases, the number of points in each observed profile, the number of points on the stellar surface, and the number of iterations. Using a VAX station, Rice and Wehlau found that typically a half to one hour was required for tabling the profiles, and two to six hours to obtain a map with fifteen to twenty iterations.

It is desirable that the final map be based on more than one line if possible. This can be done by solving the inverse problem to synthesize a portion of the spectrum containing several lines. Alternatively, separate maps may be produced from each of several lines, and then the maps are averaged. The latter method has the advantage of identifying lines which have some otherwise unrecognized problem, such as a blend with a line due to another element varying out of phase. The former method may be advantageous when the data is such that individual lines do not produce satisfactory maps. Blended lines can be used for mapping Ap stars if the components arise from the same element and the parameters of each component are known.

\section{Atomic Parameters}

Damping constants are required for all but the weakest lines in order to compute the correct line profiles. Generally accurate values for the lines of interest are not available, so various approximations have been used. If abundances are to be obtained, f-values are needed.

\section{Model Atmospheres}

To compute local line profiles, the correct model atmosphere at each point on the star should be used. Since large variations, exceeding one dex, of the abundances of the elements occur over the surfaces of some stars, we may expect that other properties of the atmosphere also vary significantly over the star.

So far, all maps have been produced using the same model atmosphere for the whole star. We know of no investigation of the effect on the resultant maps caused by ignoring the atmospheric variations produced by the abundance anomalies. This is a difficult problem for two reasons. First, appropriate model atmospheres are not available; it seems unlikely that this will be corrected soon. Even if we consider stars which show only the commonest chemical anomalies, a very extensive grid of model atmospheres would be required to cover the parameters of interest - effective temperature, gravity, and abundances. Second, the code to solve the inverse problem will become more complicated. If there is more than one element with abundance variations over the star which are sufficient to affect the atmospheric structure locally, then the inverse problem must be solved simultaneously, or by successive approximations, for each element so that the correct local model atmosphere is used. Nonetheless, it would be interesting to test this kind of effect for the simple case when only one anomalous element is responsible for modification of the atmosphere. 
It is interesting to note here that there are indications that some Ap stars have atmospheric structures which differ from those normally assumed. Shibahashi and Saio (1985) found that the observed frequencies of rapid oscillation for HD 24712 were higher than the critical frequencies which they calculated. They suggest that this discrepancy is caused either by a steeper $\mathrm{T}-\tau$ relation compared with normal A stars, or by the effects of the magnetic field. Matthews et al. (1992) found a steeper decrease of the amplitude of oscillation with wavelength than predicted for HR 3831 , based on a normal A type atmosphere. They attributed this to a larger ratio of the limb darkening coefficient in the blue than in the red for this star compared with normal $A$ stars. This also implies a steeper $\mathrm{T}-\tau$ relation for Ap stars. They found the best fit to their observations was obtained with a temperature inversion in the stellar atmosphere.

To the extent that line profiles derived from model atmospheres fail to represent the actual local line profiles on the stars, the use of analytic expressions may represent a useful simplification even when these expressions do not fully reproduce the profiles derived from model atmospheres.

\section{TESTS OF MAPPING PROGRAMS}

There are a number of solutions of the inverse problem for mapping stars in use at present. These programs may be expected to produce the same maps, given the same data. However, the computer codes are complicated, so a comparison of maps produced by different codes from the same data would be interesting. Three groups (Strassmeier et al. 1991) did independently produce temperature maps of HD 26337 using the same data. The results were quite similar but not identical; unfortunately, different graphical representations of the maps make it difficult to draw firm conclusions.

To test the ability of a solution of the inverse problem to reproduce the actual surface distribution, arbitrary artifical stellar surface distributions have been used. "Observed" spectra are calculated for a spectral line from the integral equation, assuming values for the inclination of the rotational axis, the equatorial velocity, and the limb darkening coefficient. Some noise may be added to the data, which are then used as input to the solution of the inverse problem. Since the same expressions are generally used to compute the "observed" as are used in solving the inverse problem, one must be careful not to overinterpret the results. For example, if the code makes no provision for changing the local line profile as the abundance increases, but simply increases the central depth of the line and its equivalent width, the "observed" spectra will not represent correctly the spectra that would really be observed from such a star. The solution of the inverse problem using this code could then be expected to reproduce the surface distribution better from the incorrect data than from real observations of the same artificial star where the local line profiles were correctly represented.

Various tests (Rice 1991; Rice, Wehlau and Khokhlova 1989; Hatzes, Penrod, Vogt, 1989) have shown that some information is lost in the mapping procedure. The area below and near the equator is generally poorly reproduced. Smaller features, but still above the resolution limit of the map, are not recovered. The tests also investigated the effects of errors in 
the parameters such as the inclination of the axis of rotation, the equatorial velocity, limb darkening, etc. While errors in these parameters did change the maps somewhat, no extreme sensitivity was found for small or modest errors in any of the parameters.

Comparisons of the results using Tikhonv's functional with those obtained with Maximum Entropy have been made by Rice, Wehlau, and Khokhlova (1989) and especially by Piskunov, Tuominen, and Vilhu (1990) who state "Maximizing the information entropy means that minimum correlation between different points at the stellar surface should be expected, while the use of a Tikhonov regularization functional leads to the smoothest possible solution." It is difficult to conclude which method is to be preferred when other information is not available to assist in making the choice. Fortunately, when good data are available, the penalty function has little influence on the resulting map, so the choice is not important. Figure 2 shows, on the left, the map obtained from solving the inverse problem when the artificial star had the word "RICE" written above the equator with enhanced values of $\tau$ and $\mathrm{v} \sin i=61.1 \mathrm{~km} \mathrm{~s}^{-1}$.

Piskunov, and Wehlau (1990) studied the effect of using spectra with resolutions poorer than $5 \mathrm{~km} \mathrm{~s}^{-1}$ and $\mathrm{v} \sin i$ of 15 and $30 \mathrm{~km} \mathrm{~s}^{-1}$. They concluded that useful results could be obtained, but that the maps could be misleading if some of the effects found are not taken into account. To illustrate this, Figure 2 shows an artificial stellar surface and, on the right, its reconstruction when the spectral resolution was $12 \mathrm{~km} \mathrm{~s}^{-1}$ and the value of $v \sin i$ was $15 \mathrm{~km} \mathrm{~s}^{-1}$. Since many interesting stars are too faint for us to obtain spectra at high resolution and high signal-to-noise ratios, it would be interesting to investigate further the reliability of maps obtained from spectra with more noise and low resolution.
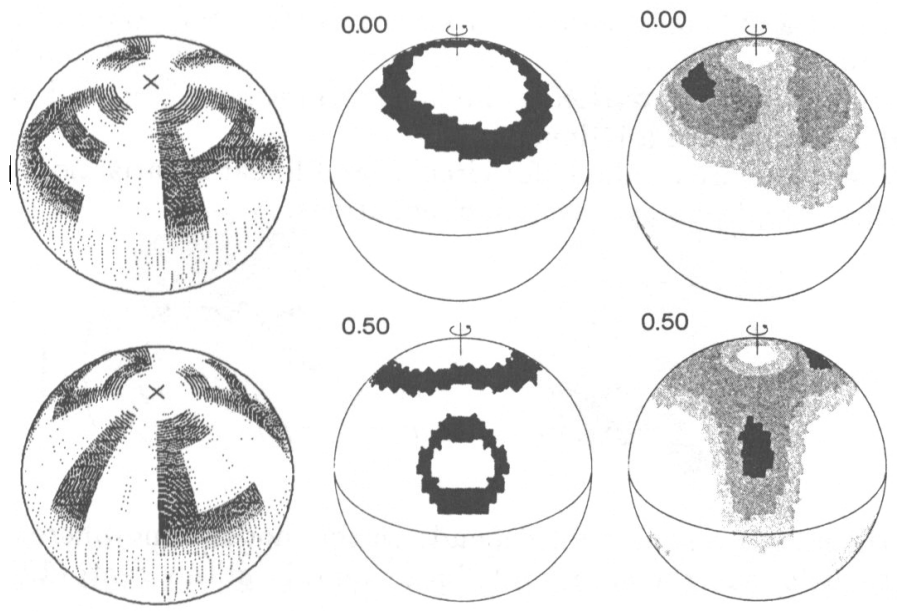

Fig. 2. The "RICE" map obtained for favorable parameters; an artificial stellar surface; and its reconstruction when the spectral resolution was $12 \mathrm{~km} \mathrm{~s}^{-1}$ and $\mathrm{v} \sin i$ was $15 \mathrm{~km} \mathrm{~s}^{-1}$. 


\section{THE MAGNETIC FIELD}

The mapping of the surfaces of Ap stars discussed above has neglected the effect of any magnetic field on the observed line profiles. For weak fields (a few hundred Gauss or less) the broadening due to the Zeeman effect is considerably smaller than the Doppler broadening when $\mathrm{v} \sin i$ is about $30 \mathrm{~km} \mathrm{~s}^{-1}$ or larger, which suggests that the effect of the magnetic field on the maps is small. On the other hand when the magnetic field is large, the Zeeman effect will dominate the line profiles of slowly rotating stars. Landstreet (see, for example, Landstreet 1988) has taken advantage of this to map the magnetic field on a number of stars. Even when the magnetic field (or the distribution of an element) varies on too small a scale to be resolved by the mapping techniques, it may still be detectable in some cases (Preston 1967).

Measurement of the profile of a line not only in intensity but also in polarization will allow the mapping of stars with significant magnetic fields and $\mathrm{v} \sin i$. If line profiles in all four Stokes' parameters have been measured, full reconstruction of the magnetic vector on the stellar surface is possible (Piskunov and Khokhlova 1984, Piskunov 1985). When both a magnetic field and an inhomogenous abundance distribution are present on a star, they should be mapped at the same time because the observed profiles in each Stokes' parameter are affected by both the magnetic field and the abundance inhomogeneities (Piskunov and Khokhlova 1983, 1984).

Line profiles at high resolution in more than one Stokes' parameter have been measured by several groups. Borra and Vaughn $(1977,1978)$ measured circular and linear polarization profiles for several stars. More recently Semel and Donati (1990) have contructed an instrument to measure I and V Stokes' parameters, and have observed a number of stars with it, including the Ap star $\epsilon$ UMa (Donati et al. 1990). Mathys (1991) has used a cassegrain spectrograph to measure $I$ and $V$ for a number of stars at a resolving power of 16,000 .

A Canadian group is building an instrument to measure the four Strokes' parameters at high resolution; a similar instrument will be used on the Nordic Optical Telescope at La Palma.

When data are available for circular and linear polarization, the equation of transfer is replaced by a system of equations:

$$
\cos \theta \frac{d}{d \tau}\left(\begin{array}{c}
I \\
Q \\
U \\
V
\end{array}\right)=\left(\begin{array}{cccc}
\eta_{0}+\eta_{I} & \eta_{Q} & \eta_{U} & \eta_{V} \\
\eta_{Q} & \eta_{0}+\eta_{I} & \rho_{V} & -\rho_{U} \\
\eta_{U} & -\rho_{V} & \eta_{0}+\eta_{I} & \rho_{Q} \\
\eta_{V} & \rho_{U} & -\rho_{Q} & \eta_{0}+\eta_{I}
\end{array}\right) \cdot\left(\begin{array}{c}
I-S \\
Q \\
U \\
V
\end{array}\right)
$$

where the matrix on the right depends on the model atmosphere and the magnetic field, and the notation follows Landi Degl'Innocenti (1976).

\section{Computational Complexities Caused by the Magnetic Field}

Consideration of the magnetic field increases considerably the amount of computation required. The magnetic field at each point on the star is specified by three quantities: the strength of the field, the inclination to the surface, and the azimuthal direction. We could attempt to proceed here as was done 
to solve equation (1) for the distribution of an element: that is, by tabling the profiles and interpolating for the required local profile. When just the abundance is considered, about a thousand profiles must be tabulated, but when the magnetic field is added, the number of profiles grows to about ten or twenty million. This approach is more costly than computing the profiles as they are needed. As as was done for equation (1) in mapping the distribution of a chemical element, we may use for solutions to equation (3) either analytic expressions or numerical ones.

To calculate the local line profiles as they are needed a fast and accurate algorithm is required. This problem has been considered by a number of authors and good solutions are available (Rees et al. ; Landi Degl'Innocenti 1976, 1987; Martin and Wickramsinghe 1979). Alternatively, an analytic representation of the solution of the equation of transfer may be used to give the local profiles in the Stokes' parameters. Analytic approximations have been given by several authors (Unno 1956, Landolfi and Degl'Innocenti 1987).

The inverse problem may be solved to yield the distribution of the magnetic field on the stellar surface using one, two three or four of the Stokes' parameter line profiles as data. Saar et al. (1992) have developed a program which maps both the magnetic field and temperaure distribution using only the I Stokes' parameter profiles for several lines with different Landé g-values. When only I profiles are available, it may also be useful to constrain the solution, as Landstreet (1988) has done by representing the field in terms of a multipole expansion.
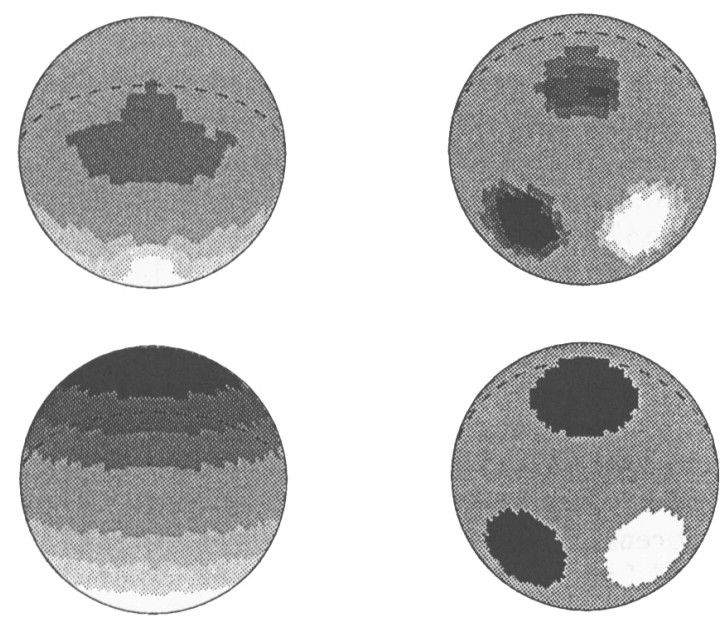

Fig. 3. Tests of mapping magnetic fields. The test stars are above. On the left the star has radial fields in three spots. On the right the star has a dipole field.

Brown et al. (1991) have developed a program to solve the inverse problem using the $I$ and $V$ line profiles as input data and the analytic approximations for the local line profiles as given by Landolfi and Landi degl'Innocenti (1982). 
For Ap stars they do not obtain maps of the distributions of the elements at the same time, and at present, ignore any effect which an inhomogeneous distribution of the elements may have on the magnetic field maps. Figure 3 shows two examples of tests which they performed in the reconstruction of magnetic fields. The test stars are shown on the top and the reconstructions below. On the left, the white spot had a radial field of $1 \mathrm{kG}$, and the black spots $-1 \mathrm{kG}$. On the right, the magnetic field is a dipole, with strength at the pole of $2 \mathrm{kG}$.

When observations of line profiles in three or four Stokes' parameters become available, it will be possible to map reliably both the distribution of an element and the surface magnetic field simultaneously. More extensive computer codes than those now in use will be required to accomplish this.

\section{ACKNOWLEDGEMENTS}

It is a pleasure to express our appreciation to Jean-Francois Donati, and Nikolai Piskunov for providing us with some of their current results for this review.

\section{REFERENCES}

Borra, E.F., and Vaughan, A.H. 1977, Ap. J., $216,462$.

Borra, E.F., and Vaughan, A.H. 1978, Ap. J., $220,924$.

Brown, S.F., Donati, J.-F., Rees, D.E., Semel, M. 1991, Astr. Ap., $250,463$.

Deutsch, A.J. 1958, in Electromagnetic Phenomena in Cosmical Physics, IAU Symp. 6, ed. B. Lehnert, Cambridge Univ. Press, Cambridge, p.209.

Falk, A.E., Wehlau, W.H. 1974, Ap. J., , 192, 137.

Goncharsky, A.G., Stepanov, V.V., Khokhlova, V.L., Yagola, A.G. 1977, Sov. Astron. 3, 147.

Goncharsky, A.G., Stepanov, V.V., Khokhlova, V.L., Yagola, A.G. 1982, Sov. Astron. 19, 576.

Hatzes, A.P., Penrod, G.D., Vogt, S.S. 1989, Ap. J., , 341, 456.

Khokhlova, V.L. 1976, Astron. Nachr., 297, 203.

Khokhlova, V.L. 1985, Sov. Sci Rev. E: Astrophys. Space Phys. Rev., 4, 99.

Landi Degl'Innocenti, E. 1987, in Numerical Radiative Transfer, ed.

W. Kalkofen. Cambridge, Cambridge University Press, 263.

Landi Degl'Innocenti, E. 1976, Astr. Ap. Suppl., 25, 379.

Landolf, M., and Landi Degl'Innocenti, E. 1982, Solar Phys. 78, 355.

Landstreet, J.D. 1988, Ap. J., 326, 967.

Martin, B., and Wickramasinghe, D.T. 1979, M.N.R.A.S., 189, 883.

Mathys, G. 1991, Astr. Ap. Suppl., 89, 121.

Matthews, J.M., Wehlau, W.H., Rice, J.B., Walker, G.A.H. 1992, in $I A U$ Collog. 137 Inside the Stars eds. W.W. Weiss and A. Baglin, in press.

Minnaert, M. 1935, it Z. Astrophys. 10, 40.

Pavlova, V.M., and Khlkhlova, V.L. 1980, Nauchnye Informatsii, Ser. Astrofiz., 43, 65.

Piskunov, N.E. 1985, Sov. Astron. Lett.,11, 18.

Piskunov, N.E., and Khokhlova, V.L. 1984, Sov. Astron. Lett.,10, 187. 
Piskunov, N.E., and Khokhlova, V.L. 1983, Sov. Astron. Lett.,9, 346.

Piskunov, N.E., Tuominen, I., Vilhu, O. 1990, Astr. Ap., 230, 363.

Piskunov, N.E., and Rice, J.B. 1992, preprint.

Piskunov, N,E., and Wehlau, W.H. 1990, Astr. Ap., 233, 497.

Preston, G.W. 1967, in The magnetic and Related Stars ed. R.C. Cameron, Baltimore: Mono Book Corp.

Pyper, D. 1969, Ap. J. Suppl., 18, No. 164.

Rees, D.E., Murphy, G.A., Durrant, C.J. 1989, Ap. J., , 339, 1093.

Rice, J.B. 1991, Astr. Ap., 245, 561.

Rice, J.B., and Wehlau, W.H. 1990, Astr. Ap., 233, 503.

Rice, J.B., and Wehlau, W.H. 1991, Astr. Ap., 246, 195.

Rice, J.B., Wehlau, W.H., Khokhlova, V.L. 1989, Astr. Ap., 208, 179.

Saar, S.H., Piskunov, N.E., Tuominen, I. 1992, preprint.

Semel, M., and Donati, J.-F. 1990, Solar Physics, 128, 227.

Donati, J.-F., Semel, M., del Toro Iniesta, J.C. 1990, Astr. Ap., 233, L17

Shibahashi, H. and Saio, H. 1985, P.A.S.Japan,37,245.

Strassmeier, K.G., Rice, J.B., Wehlau, W.H., Vogt, S.S., Hatzes, A.P., Tuominen, I., Piskunov, N.E., Hackman, T., Poutanen, M., 1991, Astr. Ap., 247, 130 .

Unno, W. 1956, Pub. A.S.P., 8, 108.

Vogt, S.S., Penrod, G.D., Hatzes, A.P. 1987, Ap. J., , 321, 496.

Wehlau, W.H., Rice, J,B., Khokhlova, V.L. 1991, Astron. Astroph. Trans.,1,55.

Wehlau, W.H., Rice, J.B., Piskunov, N.E., Khokhlova, V.L. 1982, Sov. Astron. Lett. 8, 15. 